\title{
Computed Tomography of the Brain without Contrast
}

National Cancer Institute

\section{Source}

National Cancer Institute. Computed Tomography of the Brain without Contrast. NCI

Thesaurus. Code C137897.

Computed tomography of the brain without the use of a contrast agent. 